\section{Introducción: antropología en Asturias}

IGNACIO FRADEJAS-GARCÍA ${ }^{1}$

(iD) https://orcid.org/0000-0002-2514-0654

GRAFO - Departament d'Antropologia Social i Cultural, Universitat Autònoma de Barcelona, España

\section{YOLANDA CERRA BADA ${ }^{2}$}

ASAPE (Asociación Asturiana de Antropología y Patrimonio Etnológico), España

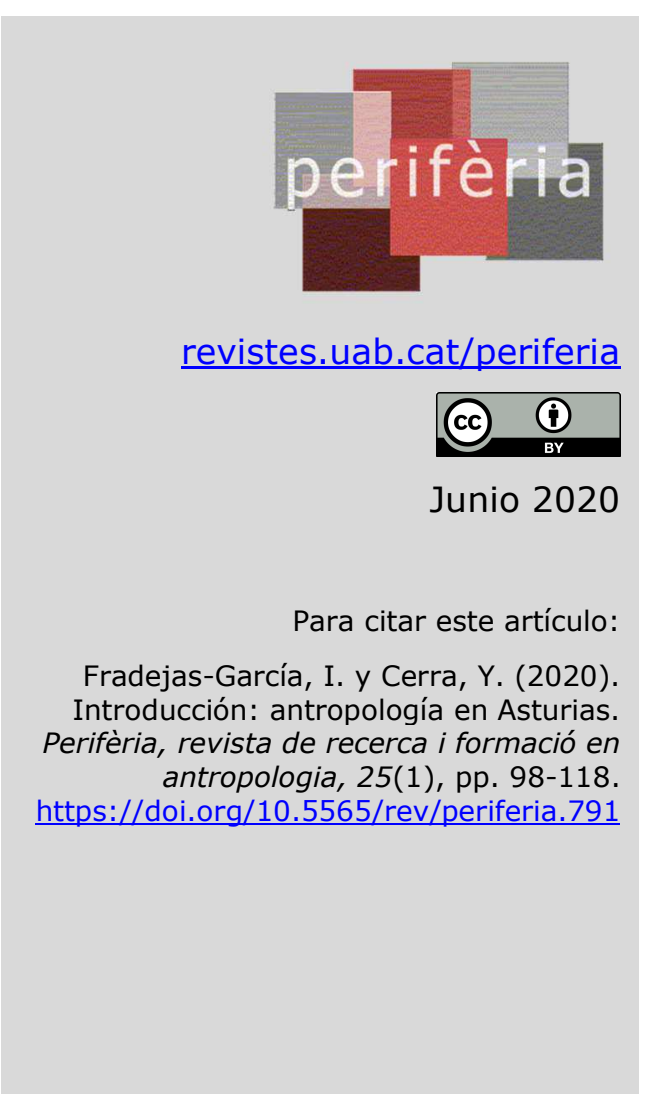

\title{
Resumen
}

Este texto y la sección especial que introduce tienen un triple objetivo. Por una parte, se intenta describir qué se ha hecho desde la antropología social y cultural en Asturias, tanto a nivel de investigación como de organización formal. Por otro lado, trata de responder a qué se está investigando hoy en día en la región. Por último, se ambiciona conectar estas investigaciones antropológicas hechas en Asturias con la teoría antropológica más actual para contribuir a crear un horizonte de institucionalización y profesionalización de la disciplina en la región.

Palabras clave: antropología en Asturias; antropología asturiana; antropología social y cultural; institucionalización; profesionalización; etnografía.

\section{Abstract: Introduction: Anthropology in Asturias}

This text introduces a special section with three objectives. First, it is an attempt to describe what has been done from social and cultural anthropology in Asturias, both at the level of research and formal organization. Second, it tries to answer what is

\footnotetext{
${ }^{1}$ Contacto: Ignacio Fradejas-García - Ignacio.Fradejas@uab.cat

${ }^{2}$ Contacto: Yolanda Cerra Bada - myolandacb@gmail.com
} 
being investigated today in the region. Finally, it aspires to connect these anthropological investigations made in Asturias with current anthropological theory, contributing to the institutionalization and professionalization of the discipline in the region.

Key words: Anthropology in Asturias; Asturian Anthropology; Social and Cultural Anthropology; institutionalization; professionalization; ethnography.

\section{Los porqués de una antropología en Asturias}

La antropología social y cultural es una ciencia social institucionalizada con características particulares desde principios del S.XX, aunque su presencia institucional en Asturias no se ha formalizado hasta principios del S.XXI. La carencia de instituciones, asociaciones, departamentos y grupos de investigación dedicados a la antropología en Asturias tiene una relación directa con la falta de profesionalización de la disciplina, como pasa en otras regiones españolas (Abad, 2011) ${ }^{3}$. Sin embargo, el terreno antropológico en esta zona el norte de España está plagado de numerosas iniciativas individuales y pequeños grupos que han compensado el relativo desinterés institucional de y por la disciplina.

Esta introducción y el conjunto de textos que componen la sección especial "Antropología en Asturias" tratan de responder a las siguientes preguntas: ¿es posible hablar de una antropología en/de/para Asturias?, ¿qué investigaciones se están llevando a cabo y cómo se conectan con las necesidades de la gente y con las teorías antropológicas más actuales a nivel internacional? Por tanto, nuestros objetivos generales son describir qué se ha hecho desde la antropología en esta comunidad autónoma española, tanto a nivel de investigación como de organización formal y, más importante, queremos saber qué se está investigando hoy en día en la región.

Es necesario puntualizar que en este texto no consideramos Asturias un objeto de estudio sino un territorio definido de forma política y administrativa mediante un proceso histórico, unas dinámicas culturales y unas relaciones sociales que la

\footnotetext{
${ }^{3}$ Abad (2011) no pudo encontrar en Asturias grupos de investigación en antropología (ni antropólogos o antropólogas trabajando en otros grupos).
} 
desbordan tanto en el tiempo como en el espacio. El Principado de Asturias, como se denomina oficialmente, es una comunidad autónoma española situada en el norte de la península ibérica. Está habitada por poco más de un millón de personas que se concentran en el área metropolitana formada por las ciudades de Oviedo, Gijón, Avilés, Mieres, Langreo y Siero. Su carácter de comunidad histórica, su cultura y su lengua (el asturiano, no reconocido oficialmente), así como su orografía montañosa bañada por el atlántico y su situación periférica con respecto a los centros políticos y productivos, le confieren un carácter particular (a veces exotizado) dentro de las fronteras administrativas del Estado español.

Partiendo de esta forma abierta de entender la construcción social y cultural del lugar de estudio, pretendemos entender las conexiones concretas que ocurren a múltiples escalas dentro de procesos históricos de acumulación y desposesión capitalista en Asturias. La actual pandemia global por el COVID19 es una trance más dentro de una época marcada por crisis globales de los sistemas económicos, identitarios y ecológicos (Eriksen, 2016) y explicada mediante teorías macrosociales que destacan la globalización, la fluidez y la hipermovilidad de personas, capitales, servicios y conocimientos. En este contexto de incertidumbre es más necesario que nunca un conocimiento situado y local de las prácticas sociales y culturales, que pueden abordarse mediante el estudio de áreas y regiones culturales (Mintz, 1998). Así, estamos de acuerdo con Susana Narotzky cuando propone una antropología regional que acuda al realismo etnográfico y abandone la acumulación de estudios de lugares como cosas transhistóricas desconectadas de problemáticas generales: no hay etnografía sin teoría ni antropología sin descripción etnográfica (Narotzky, 2001).

Paralelamente, no podemos dar la espalda a procesos globales y transnacionales que tienen un impacto directo en la cotidianeidad de las personas que estudiamos. Las propuestas para realizar una antropología que conecte lo local y lo global a varias niveles (e.g. Tsing, 2005) trata de evitar el análisis de estados y naciones delimitados por fronteras políticas y/ administrativas como contenedores sociales y culturales homogéneos (Wimmer \& Glick Schiller, 2002). Estos enfoques multiescalares pueden ser utilizados para estudiar viejas y nuevas temáticas que abordan la investigación desde marcos más especializados. Como ejemplo de esta antropología heterogénea, se pueden destacar las redes autoformadas por antropólogos y antropólogas de la 
"European Association of Social Anthropologists"4 (EASA), el referente asociativo a nivel europeo de la disciplina, que se enfocan en temas tan diversos como antropología (de/l): arte, movilidad, niñez y juventud, economía, comida, género y sexualidad, humanitarismo, trabajo, derecho leyes y gobernanza, raza y etnicidad, religión, seguridad, estado, experimentación colaborativa, desastre y crisis, europeísmo, mediterraneísmo, africanismo, queer, psicología, medios, medicina, paz y conflicto, peregrinaje, urbana, visual, etc.

Este listado nos acerca a un doble propósito de esta sección especial. El primer propósito es conocer los vínculos de las investigaciones actuales con temas relevantes para Asturias, como pueden ser la gobernanza de/en la periferia, la gestión del patrimonio material e inmaterial, la oficialización del asturiano, las economías alternativas, las políticas de austeridad, el envejecimiento de la población, el vaciamiento rural, los movimientos sociales, la desindustrialización, la fuga de cerebros, la precariedad laboral o el futuro del trabajo. El segundo propósito es conectar estos estudios con las problemáticas antropológicas actuales que se ocupan de temas globales como la emergencia climática, el género y las sexualidades, la (in)movilidad, la migración y el transnacionalismo, las políticas neoliberales, el auge de los nacionalismos y los populismos, el crecimiento y la externalización de las fronteras, las relaciones entre humanos y no-humanos, el post-colonianismo y la decolonialidad, o las dificultades ético-metodológicas del quehacer antropológico. Ambos propósitos plantean enfoques y temáticas que, sin ánimo de exhaustividad, nos ayudan a pensar en problemas sociales no abordados hasta ahora de forma sistemática en Asturias mediante y desde la antropología social y cultural.

\section{Una historia de la antropología asturiana a través de sus autores}

La antropología asturiana, dejando atrás a precedentes como Jovellanos y otros, la inaugura una desconocida, Purificación Viyao Valdés (1892-1934). Alumna de Luis de Hoyos en la Escuela Superior de Magisterio, escribe bajo su dirección su trabajo fin de carrera titulado Datos antropo-etnográficos de la parte oriental de Asturias: el

\footnotetext{
${ }^{4}$ https://www.easaonline.org/networks/
} 
hombre y el medio (1920), el cual permaneció inédito casi noventa años 5 . Tras esta antropología española de influencia naturalista que recoge muchas líneas de trabajo y que todavía se está definiendo, las nuevas corrientes teóricas anglosajonas inauguran inmediatamente la etapa clásica de la antropología. La guerra civil y las primeras décadas de la dictadura paralizarán cualquier intento de construcción de la disciplina y habrá que esperar con mucha paciencia hasta notar la muy escasa presencia de algún antropólogo o antropóloga en Asturias.

Será el alicantino Ramón Valdés del Toro $(1930-2011)^{6}$, recién llegado al instituto de Tapia de Casariego como profesor de Geografía e Historia en 1959, quien inicie la nueva etapa de la moderna antropología social y cultural en Asturias. Ese mismo año comienza su trabajo de campo, cuyo resultado, orientado por la ecología cultural, publicará años más tarde bajo el título "Ecología y trabajo, fiestas y dieta en un concejo del occidente astur" (Valdés, 1976). En el curso 1965-66 se incorpora a la Universidad de Oviedo como profesor de Etnología dentro de la Sección de Historia de la Facultad de Filosofía y Letras, siendo nombrado el curso siguiente profesor adjunto de Antropología y Etnología, donde seguirá hasta 1971.

Los tímidos intentos de institucionalización de esta disciplina en Asturias van de la mano de Valdés, pero un incidente estudiantil y posteriores desavenencias con el filósofo Gustavo Bueno, quien le había llevado a la universidad y que renuncia a continuar con la dirección de su tesis doctoral, lo conducirá finalmente a la Universidad Autónoma de Barcelona7. Se da como fecha de la institucionalización de la antropología española el año de 1971 cuando Esteva Fábregat obtiene su cátedra en dicha universidad y se forma el departamento. A este se incorpora casi inmediatamente Valdés del Toro. Alberto Cardín y Aurora González Echevarría, licenciados en Oviedo, siguen la senda de Valdés hacia Barcelona. Este sucede en la cátedra a Esteva y finaliza sus días como catedrático emérito.

\footnotetext{
${ }^{5}$ Se publicó junto con otra memoria fin de carrera: Viyao Valdés, P. y Martín-Ayuso Navarro, R. (2007). Próximamente, para conmemorar el centenario de su publicación, entrará en prensa la reedición del trabajo de la primera, con estudio introductorio de Yolanda Cerra Bada.

6 En este mismo número de Perifèria se publica en formato de 'Reimpresión' la conferencia jubilar de Ramón Valdés del Toro titulada "Mi definición de religión"

7 Para ahondar en estos eventos véase el texto de Aurora González Echevarria en esta misma sección especial, titulado "Reflexiones sobre los inicios de la antropología en Asturias. De nativa a antropóloga".
} 
En 1965 llegan a Asturias a realizar trabajo de campo, tras su etapa africanista, un norteamericano descendiente de asturianos, James W. Fernández, y su esposa Renate Lellep, quienes se instalan en Felechosa (Aller) y más tarde en los concejos de Onís y Cangas de Onís. Ella publicará un trabajo sobre el bocio en las cuencas mineras, "A Simple Matter of Salt: An Ethnography of Nutritional Deficiency in Spain" (Lellep, 1990), y él, catedrático emérito de Antropología en la Universidad de Chicago, trabaja sobre la teoría de la configuración del pensamiento y acción social, lo que dará lugar a obras como "Campos léxicos y vida cultural n'Asturies" (Fernández, 1996), "En el dominio del tropo. Imaginación figurativa y vida social en España" (Fernández \& Velasco, 2011), a las que se añade "El carácter asturiano y otros enigmas e improntas de las identidades hispánicas" (Fernández, 2009).

En 1969, María Cátedra llega a Asturias para realizar el trabajo de campo de su tesis doctoral en las brañas vaqueiras. La que luego será catedrática emérita de Antropología Social de la Universidad Complutense, realizó sus doctorados en esta Universidad y en la de Pennsylvania con sendas investigaciones realizadas entre los vaqueiros de alzada, que serán publicadas en los libros siguientes: "Death as a Cultural Process. The Vaqueiros de Alzada, Spain: A dissertation in Anthropology" (Cátedra, 1986), "La muerte y otros mundos. enfermedad, suicidio, muerte y más allá entre los vaqueiros de alzada" (Cátedra, 1988) y "La vida y el mundo de los vaqueiros de alzada" (Cátedra, 1989), así como en otros artículos.

En 1974 se incorpora el asturiano José Luis García García ${ }^{8}$ al trabajo de campo, primero en Villanueva de Oscos y posteriormente en el concejo de Aller. García, catedrático emérito de la Universidad Complutense de Madrid, estudió aquí la antropología del espacio, el análisis del discurso, los rituales o la actividad minera. En su primera obra, "Antropología del territorio" (García, 1976), dedica gran parte al análisis territorial de dos comunidades españolas muy distintas, situadas ambas en Asturias: el poblado minero de Bustiello (Mieres) y Villanueva de Oscos, en los límites con Galicia. Es coautor de "Rituales y proceso social. Estudio comparativo en cinco zonas españolas" (García, Velasco, \& López, 1991), donde analiza esta cuestión en Los Oscos. Respecto a la actividad minera, escribió "Prácticas paternalistas. Un estudio antropológico sobre los mineros asturianos" (García, 1996) y dirigió

\footnotetext{
${ }^{8}$ Se publica en esta sección un obituario de José Luis García García, recientemente fallecido, titulado "El antropólogo José Luis García y Asturias. En memoria" y escrito por Juaco López.
} 
proyectos de investigación sobre las prejubilaciones y la minería, recogidos en obras de conjunto como "Los últimos mineros. Un estudio antropológico sobre la minería en España" (García et al., 2002), donde escribe "Mineros alleranos: de los diferentes segmentos sociales a la construcción de la comunidad", y la compilación "Prejubilados españoles. Ajustes y resistencias ante las políticas públicas" donde contribuye con "La intervención política y la construcción de las categorías colectivas. La prejubilación de los mineros como problema social" (García, 2009).

Juan Oliver Sánchez Fernández, asturiano, también profesor de la Complutense, inicia su trabajo de campo con los vaqueiros de alzada de Somiedo, después continúa con la pesca y la minería del carbón, investigaciones que vieron la luz en obras como "Ecología y estrategias sociales de los pescadores de Cudillero" (Sánchez, 1992) o "Trabajo, política e ideología en una cuenca minera" (Sánchez, 2004). Marie José Devillard, catedrática en la Universidad Complutense, hace trabajo de campo en Langreo y trabaja con García la minería y las prejubilaciones. Escribió "«Yé una cadena». Minería y estrategias de reproducción social" (Devillard, 1997) y es coautora junto con García y otros autores de "Los últimos mineros. Un estudio antropológico sobre la minería en España" donde publica "Crisis, realidades y representaciones del espacio minero langreano en vísperas del siglo XXI" (Devillard, 2002). La asturiana Nieves Herrero Pérez, profesora titular de Antropología en la Universidad de Santiago de Compostela, ha escrito algunos artículos sobre un pueblo cercano a su localidad natal como "Las glorietas para la Sacramental de Naves (Asturias): marcadores rituales del territorio" (Herrero, 2014). Por último, Nancy Konvalinka, catedrática de la UNED con relaciones de parentesco en Asturias, hizo entrevistas de campo en esta región sobre la gestación subrogada.

Todas estas personas constituyen un grupo que desarrolla su carrera académica fuera de Asturias, principalmente en la Universidad Complutense de Madrid. Los nativos de Asturias o quienes tienen antecedentes familiares y parentesco se acercan de manera puntual o con cierta continuidad a esta región para realizar sus investigaciones de campo, con la excepción hecha de José Luis García que desde sus inicios y durante toda su carrera hizo etnografía en su tierra natal. La mayoría realizan estudios de comunidades campesinas, vaqueiras, pescadoras y mineras.

Frente a este grupo, con anclaje definitivo en la universidad, se alza otro que se mantiene profesionalmente fuera de la antropología académica. Se trata de dos profesores de enseñanza secundaria de humanidades, uno de los cuales tiene 
docencia ocasional en la universidad, así como de un profesor universitario que imparte docencia en disciplinas afines a la antropología.

Adolfo García Martínez empieza su trabajo de campo a mediados de los años 70 en las brañas vaqueiras. Formado en Francia, es licenciado en Filosofía por la Universidad Libre de Lyon y en Sociología por el Institut de Sociologie de Lyon; también obtiene la titulación en Filosofía y Letras por la Universidad de Valencia. Lee su tesis doctoral en la universidad de Oviedo que se publica con el título "Los vaqueiros de alzada de Asturias. Un estudio histórico-antropológico" (García, 1988). Catedrático de Filosofía de instituto, impartió docencia en Antropología en las universidades de Valladolid y de Oviedo, así como en el máster de Historia y Análisis sociocultural de esta última universidad, donde dirigió trabajos de investigación y tesis doctorales. También impartió esta asignatura en la Facultad de Derecho, en la Escuela de Trabajo Social y en la de Magisterio y fue profesor tutor en la UNED hasta su jubilación. Se da la circunstancia de que, a finales de los 70, estuvo cuatro años en la recién creada Facultad de Filosofía asturiana, creyendo en la promesa de que se encargaría de la asignatura de Antropología, pero, al no ver perspectivas de futuro, acabó opositando a enseñanza media. Autor muy fecundo, es destacable su obra en dos volúmenes "Antropología de Asturias I: la cultura tradicional, patrimonio de futuro" (García, 2008) y "Antropología de Asturias II. El cambio: la imagen invertida del otro" (García, 2011). También merece la pena ser destacada su contribución a la antropología aplicada, por medio de la puesta en marcha del Ecomuseo de Somiedo y el Ecomuseo del pan en Villanueva de Oscos.

Roberto González-Quevedo, catedrático de Filosofía de instituto, realizó trabajo de campo para su tesis doctoral en Palacios del Sil, su lugar de procedencia, una comarca leonesa que comparte rasgos culturales con el suroccidente de Asturias. Asentado definitivamente en Asturias, dirige sus investigaciones a esta comunidad autónoma y publica, entre otras obras, "Antropología social y cultural de Asturias" (González-Quevedo, 2002a), "Aspeutos de la muerte n'Asturies" (González-Quevedo, 2002b) y "La cultura asturiana" (González-Quevedo, 2010). Desde 1990 dirige "Cultures. Revista Asturiana de Cultura", editada por la Academia de la Llingua Asturiana, que en sus primeros años albergaba artículos de antropólogos nacionales y extranjeros. 
Por último, cabe citar a Eloy Gómez Pellón, licenciado en Filosofía y Letras y en Derecho, que realizó trabajo de campo en el concejo de Llanes para su tesis doctoral leída en la universidad asturiana y publicada con el título "Vida tradicional y proceso de cambio en un valle del oriente asturiano: estudio antropológico del Valle de Ardisana" (Gómez, 1990). También escribió una obra de síntesis, "Las mascaradas de invierno en Asturias. Una perspectiva antropológica" (Gómez, 1993). Desde la Facultad de Derecho de la Universidad de Oviedo prosigue su carrera en la de Cantabria donde actualmente es catedrático de Antropología.

Hay otro profesor, José Manuel Gómez-Tabanera García, difícilmente clasificable, que no pertenece a ninguno de estos dos grupos y que cabría mencionar aquí. Nacido en Barcelona, con familia de ascendencia asturiana, estudió Derecho y Filosofía y Letras en Madrid. Publicó su tesis doctoral, "Teoría e Historia de la Etnología", en 1953 y dos años más tarde, su memoria de licenciatura, "Estudios sobre el totemismo y su significación en la cuenca del Mediterráneo antiguo y la España primitiva". Fue profesor ayudante de Prehistoria e Historia Antigua en la Complutense y profesor interino de Prehistoria y otras asignaturas, como Etnología, en la Universidad de Oviedo, donde permaneció desde 1969 a 1978. Escritor muy prolífico, divulgador y editor, su posición acomodada le permitió discurrir al margen de la institución académica.

El caso particular de Asturias, donde, al contrario que en otras autonomías, no se implantan los estudios de antropología y, por tanto, no se cuenta con el respaldo académico, hay muchos otros trabajos de carácter etnográfico, entre los que destacan los realizados de manera individual o conjunta por Armando Graña y Juaco López, declarados deudores de Caro Baroja. Estos, con inicial formación universitaria en Geografía e Historia, especialidad en Prehistoria y Etnologia, en la Universidad Complutense, publicaron desde los años 80 libros centrados en el carácter formal de la cultura material. En concreto, estudiaron los hórreos y paneras (Graña \& López, 1983); ferrerías, mazos y fraguas (López, Quirós, \& Graña, 1998); o la arquitectura de cubierta vegetal (Graña \& López, 2007). Graña realiza su tesis sobre carpintería y arte popular en los hórreos y López sobre las abejas, la miel y la cera (López, 1994). Este último, director del Muséu del Pueblu d'Asturies, tiene también obra sobre festividades, fotografía, emigración y escritura popular. 
El fallido intento de institucionalización en el departamento de Filosofía de la Universidad de Oviedo y la diáspora en la Universitar Autònoma de Barcelona, la llegada de investigadores extranjeros y nacionales, en especial de la Universidad Complutense de Madrid, a realizar trabajo de campo, en algún caso buscando el exotismo local, así como la orfandad académica de los que trabajan dentro de Asturias dirigiendo sus investigaciones a la propia comunidad, marcan la antropología social y cultural asturiana de las tres últimas décadas del siglo XX.

Una vez iniciado el siglo XXI, se perfila un nuevo escenario con nuevos actores. Un convenio de colaboración firmado entre el Departamento de Antropología Social de la Universidad Complutense de Madrid y el Muséu del Pueblu d'Asturies, que descansa en la relación entre José Luis García y Juaco López ${ }^{9}$, va a posibilitar la realización de investigaciones en Asturias, así como la publicación aquí de sus obras. Cabe mencionar a Elisabeth Lorenzi Fernández y Jorge Moraga Reyes, que estudian la reciprocidad en dos campos, la primera, en el ámbito rural, con el reestudio del trabajo de Valdés del Toro, publicado en "¿Bótoche unha mao? La evolución de las relaciones de reciprocidad campesinas en Tapia de Casariego" (Lorenzi, 2004); el segundo, en una comunidad inmigrante urbana, publicado como "Chinos en Asturias. La reciprocidad en el imperio del cálculo" (Moraga, 2010). También se puede citar otra publicación nacida de este ambiente de colaboración, el libro de Rocío PérezSutil del Cueto "Cabranes: de la casería a la casa de huéspedes" (Pérez-Sutil, 2019). En paralelo a ese convenio, se produce la implantación de la licenciatura en Antropología Social y Cultural de la UNED (Universidad Nacional de Educación a Distancia), que tiene lugar en el curso 2003-04, seguido luego del Grado, lo que hace posible, por fin, realizar estos estudios en Asturias. Allí convergen los primeros años no pocas personas vocacionales, que se habían orientado a otros perfiles profesionales. La orfandad académica a que obliga la Universidad de Oviedo, que en la actualidad no tiene ni siquiera una asignatura de antropología general, conduce a que aquí se dé cierta relevancia a la UNED. El animador en aquellos primeros años y hasta su jubilación fue Adolfo García Martínez, profesor tutor de varias asignaturas.

\footnotetext{
${ }^{9}$ Véase el texto de Juaco López publicado en este mismo número para conocer en detalle este convenio.
} 
En ese mismo ambiente de la universidad a distancia se forja la ASAPE (Asociación Asturiana de Antropología y Patrimonio Etnológico), fundada en 2014 con el objetivo de defender la disciplina y la profesión, con Adolfo García Martínez como socio de honor, y que aglutina a una nueva generación de antropólogas y antropólogos. Entre estas encontramos, sin ánimo de exhaustividad, a dos cofundadoras de la asociación, Yolanda Cerra Bada y Cristina Cantero Fernández, a dos personas que contribuyen en esta sección especial, Heriberto Gutiérrez y Carlos Suari, así como al etnomusicólogo Llorián García Flórez (música y políticas de la escucha en la Asturias postfranquista), a la antropóloga Noelia García Rodríguez (cambios ambientales, sociales y económicos derivados de la reclasificación de parques naturales) y al antropólogo Xuan de Con Redondo (sidra y patrimonio). También forman parte de esta generación personas de origen asturiano que están realizando su carrera académica fuera de la región, aunque mantienen los vínculos, como Montserrat Cañedo Rodríguez (UNED), Óscar López Catalán (UAB) o Ignacio Fradejas-García $(U A B)$, aunque seguro que hay muchas otras.

Esta nueva remesa empieza tímidamente a hacer visible una disciplina que, desde sus inicios en el departamento de Filosofía, ha ido perdiendo presencia en la universidad ovetense, haciendo excepción del apoyo que proporcionan ahora los historiadores sociales en el máster de Historia y Análisis sociocultural, contexto donde se trae profesorado visitante de antropología y se hacen Trabajos Fin de Máster y alguna tesis doctoral de orientación antropológica.

Yolanda Cerra Bada, licenciada en Filología y en Antropología Social y Cultural, fue profesora de Lengua y Literatura de instituto y profesora tutora en la UNED. Etnógrafa de la danza primero, con "Bailes y danzas tradicionales en Asturias" (Cerra, 1991), realiza después su trabajo de campo principalmente en la zona oriental de Asturias. Se especializa en la etnohistoria del sistema dual-triádico de la villa de Llanes, con sociabilidad festiva asociada al territorio, y la reelaboración de tradiciones rurales, que plasma en diversos trabajos como, por ejemplo, "La invención y la recuperación de los bailes tradicionales" (Cerra, 2008) o "Conservar mientras se da: aldeanas que ofrecen ramos" (Cerra, 2015), así como en su tesis de próxima publicación.

Cristina Cantero Fernández, licenciada en Geografía e Historia, con estudios en antropología, realiza trabajo de campo en Asturias sobre diversos temas que en general responden a la construcción de discursos e imágenes socioculturales. Es 
autora de "Etnohistoria del cotu de Curiel (Cenero, Xixón)" (Cantero, 2003) y "El agua en la vida cotidiana. Fuentes, lavaderos y abrevaderos de Asturias" (Cantero, 2015). Ha plasmado su labor museográfica en varias instituciones como el Museo de Quirós y Comarca y realizado numerosos catálogos de patrimonio etnológico, así como investigaciones e informes técnicos acerca de los vaqueiros de alzada, el derecho consuetudinario o los santuarios. Muchos de estos trabajos son encargos de la administración, como el "Documento-marco de recomendaciones sobre la gestión de bienes patrimoniales de carácter etnológico: hórreos y paneras" (Cantero, 2019). Fe Santoveña Zapatero, licenciada en Geografía e Historia y en Antropología Social y Cultural, trabaja sobre la construcción social de la identidad a través del vestido. Realiza para su tesis una investigación sobre la construcción del traje regional asturiano basada en fuentes documentales fotográficas, que será publicada bajo el título "Traje tradicional. Indumentaria popular y construcción social del cuerpo en Asturias (1860-1920)" (Santoveña, 2018). También es autora, entre otras obras, del libro "Vestidos de asturianos. Ciento cincuenta años de fotografía e indumentaria en Asturias" (Santoveña, 2013). Además, contribuye en esta sección especial con un artículo titulado "En la encrucijada. Traje tradicional asturiano, identidad y transformaciones sociales en los inicios del siglo XXI".

En cuanto al trabajo asociativo de ASAPE, este permite traer a Asturias a profesionales de prestigio para participar en ciclos de conferencias, así como publicar sus intervenciones. Por ejemplo, el libro "Los inicios de la antropología en Asturias" (Fernández, Cátedra, \& García, 2016) recoge las de James W. Fernández, María Cátedra y José Luis García en un ciclo realizado conjuntamente por el Muséu del Pueblu d'Asturies y ASAPE. Ese trabajo también permite el asesoramiento a la administración sobre patrimonio etnológico, labor que realiza Yolanda Cerra Bada, integrante de la Comisión de Patrimonio Etnográfico junto con Juaco López Álvarez; a esta labor se suman Cristina Cantero Fernández y Xuan de Con Redondo, quien a la vez sucede en la UNED a García Martínez. Todas estas colaboraciones, así como las memorias para la declaración BIC del corri-corri (Cerra, 2017), sidros y comedies (Cantero, 2018) o, de esta última, conjunto texu-santuario, así como la candidatura de la piedra seca a Patrimonio Cultural Inmaterial de la Humanidad, que son trabajos 
de investigación ${ }^{10}$, permiten ir introduciendo la perspectiva antropológica en ese ámbito administrativo.

Este acervo de producción etnográfica, etnológica y antropológica ${ }^{11}$ llevado a cabo por autores a título individual, o motivado por algunas iniciativas formales, puede llevarnos a concluir que existe una antropología asturiana, referida tanto al espacio como al desarrollo de esta y a sus características, o a la formación de personas dedicadas a la antropología y a su profesionalización. Sin embargo, queda un largo camino por recorrer hasta poder hablar en estos términos, tal y como ha quedado patente. Además, este listado no es exhaustivo ni trata de crear un canon, al contrario, conocemos sus limitaciones tanto referenciales como disciplinares. Un paso necesario sería aglutinar en un mismo documento toda la bibliografía etnológica, etnográfica y antropológica referida o realizada en Asturias. Podría ser similar a la organizada en los años 1990 por Joan Prat a nivel español, que agrupó para su consulta 7.721 entradas de 604 autores (Prat, 1999), aunque aquí pensando en ir actualizando a medida que aparezcan nuevas (o viejas) publicaciones. Por último, queremos destacar que las fronteras disciplinarias son porosas $y$, por ejemplo, el departamento de sociología en la Universidad de Oviedo investiga temáticas que son también muy antropológicas como la alimentación, el trabajo o el estado del bienestar. En un contexto global de creciente interdisciplinariedad, pensamos que la antropología puede aportar un enfoque y una metodología distintiva y abierta a los problemas sociales que nos rodean.

\section{Hacia una 'antropología asturiana'}

En el contexto investigador y académico actual, la financiación es indispensable para realizar investigación de calidad y poder favorecer la producción de conocimiento que aborde problemáticas sociales. En Asturias, hay tres factores que se retroalimentan para dificultar la atracción de estos fondos de investigación: institucionalización todavía incipiente, falta de profesionalización y carencia de grupos de investigación.

\footnotetext{
10 Tras su exposición pública en la web institucional www.asturias.es, una vez realizada la declaración del bien, los trabajos se hallan en depósito en Dirección General de Patrimonio Cultural.
} 
Por ahora, este texto y los artículos que componen esta sección especial tratan de caminar hacia ese horizonte de institucionalización. La sección se compone de seis textos de muy variada condición: dos artículos, dos textos de apuntes etnográficos, una necrológica y un epílogo. El primero de los artículos es autoría de Heriberto Gutiérrez y se titula "La represión franquista sobre las mujeres en Asturias: reconstruyendo la posmemoria con relatos familiares". El texto nos acerca los relatos de la represión franquista en los valles mineros asturianos, donde varios tipos de violencia sobre las mujeres coexistieron dejando tras de sí una memoria llena de omisiones en forma de parches y agujeros. Estos hechos se conectan con otros semejantes que han ocurrido durante la guerra de los Balcanes o en el genocidio ruandés, para mostrar cómo opera la desmemoria a nivel estructural y personal, dando paso a la construcción de la posmemoria personal y familiar. La guerra y la represión pueden parecer cosas del pasado, sin embargo, la violencia específica contra las mujeres sigue ocurriendo en conflictos y postconflictos actuales, como en Siria o Yemen, por citar solo un par de ejemplos.

El segundo texto opera a un nivel distinto, en las arenas sociales y culturales de la región, que van añadiendo capas de complejidad a fenómenos muy visibles de carácter identitario, como son los trajes tradicionales. El texto de Fe Santoveña se titula "En la encrucijada. Traje tradicional asturiano, identidad y transformaciones sociales en los inicios del siglo XXI". La autora nos lleva a transitar por el proceso histórico que ha derivado en los distintos cánones del traje regional asturiano. A través de las diferentes etapas que operan como arenas de imposición de estéticas y modas, la autora presenta las batallas y modas políticas que han desembocado en una posmodernidad que utiliza a su antojo aquello que le sirve en cada momento.

Esta puesta en escena del asturianismo conecta con el texto de Carlos Suari, titulado "El baile tradicional asturiano como práctica étnica performativa en la primera cuarentena global". Los apuntes etnográficos del autor nos aproximan, mediante una elaborada etnografía virtual apoyada en su trabajo de campo previo, a los eventos online cuasibailables producidos durante el confinamiento por la pandemia del COVID 19 de la primavera del año 2020. El autor se sumerge en las prácticas y los discursos de escenas bailables en los hogares, para revelar la emergencia de elementos étnicos y performativos de un nuevo asturianismo que conecta glocalmente redes grupales y significados compartidos. 
Adolfo García, por su parte, acude a la etnografía clásica y nos regala un texto titulado "Etnografía de la pala del pan: de la casa asturiana a la dualidad del patrimonio tangible-intangible". El texto viaja desde el caserío asturiano en el que el pan era un elemento central de la vida campesina, al museo etnográfico donde el objeto pierde el olor del contexto y el sabor de las relaciones sociales y productivas que lo dotan de significado. Además, Adolfo comparte fotos de incalculable valor, fruto de una vida dedicada a la etnografía en Asturias.

Por último, tenemos dos textos en clave de recuerdo y nostalgia. El primero es un obituario que ha escrito Juaco López para acercarnos la figura de su colega y antropólogo asturiano José Luis García García, recientemente fallecido. Esto entronca con el fallecimiento reciente de otra figura clave en la etnografía asturiana, Pepe'l Ferreiro, etnógrafo asturiano autodidacta y fundador Muséu Etnográficu de Grandas de Salime. Descansen en paz.

El segundo texto es un epílogo que condensa a modo de obra teatral en tres actos toda una vida antropológica. Su autora es Aurora González Echevarría, quien nos obsequia con una pequeña joya titulada "Reflexiones sobre los inicios de la antropología en Asturias. De nativa a antropóloga", donde desgrana los eventos que la condujeron a ella y a su mentor, Ramón Valdés, de Asturias a Barcelona para poner en marcha el departamento de Antropología Social y Cultural de la Universitat Autónoma de Barcelona (dónde posteriormente se funda también la revista Perifèria). El último acto es un brillante epílogo intelectual que pone de relieve la necesidad de realizar comparaciones transculturales y una conceptualización etnográfica rigurosa.

Queremos destacar que, sin dejar de mirar a lo que ya se hace y se ha realizado, Asturias puede ser un terreno muy fértil para investigaciones novedosas que se conectan con fenómenos y problemas actuales de carácter internacional. Como ejemplo, queremos destacar un reciente artículo del antropólogo José Antonio Cortés Vázquez (2020), quien fue invitado a participar en el año 2017 como experto en la Junta General del Principado de Asturias para debatir una ley sobre la Protección de Espacios Naturales del Principado de Asturias. Fruto de aquellos debates y de sus estudios etnográficos previos sobre la gestión y gobernanza de parques naturales, el texto conecta fenómenos sociales locales con procesos globales como la desposesión neoliberal, la crisis climática y el advenimiento de partidos y postulados de extrema derecha (Cortes-Vazquez, 2020). El texto de Cortes-Vazquez es un modelo a seguir 
del tipo de antropología básica y aplicada, con potencial para informar políticas públicas, que al mismo tiempo produce conocimiento de calidad publicado en revistas académicas de primer nivel internacional.

Para finalizar, queremos retomar la historia que pudo fundar hace 50 años una antropología asturiana. Gustavo Bueno y Ramón Valdés querían hacer una elipse, al modo del círculo de Viena, aunque parece no quedó claro en qué consistía (Hidalgo, 2017). Sus desavenencias acabaron en la migración de Valdés para crear el departamento de antropología de la Universitat Autónoma de Barcelona; la elipse no pasó de parábola y el círculo no se cerró nunca. Por ello, con este texto no tratamos de recuperar un tiempo pasado ni conectar espirales perdidas, sino de animar a generar nuevas trayectorias antropológicas que no se vean interrumpidas y puedan desarrollarse en Asturias respetando el camino ya realizado, pero yendo más allá de las fuerzas centrífugas y centrípetas que algunos académicos juegan en la conformación de las disciplinas y los cánones. Como dijo José Luis Molina respecto de Ramón Valdés, su mentor: "Siempre recordaré sus críticas amables, pero despiadadas al mismo tiempo. Y es que Ramón solía repetir que su único compromiso era con la verdad y no con nosotros." (Molina, 2011: 332).

\section{Bibliografía}

Abad, J. S. (2011). Panorámica general de los grupos de investigación en Antropología Social en el Estado español. Revista de Antropología Social, 20. https://doi.org/10.5209/rev_RASO.2011.v20.36236

Cantero, C. (2003). Etnohistoria del Cotu de Curiel (Cenero, Xixón). Gijón: Fundación Municipal de Cultura, Educación y Universidad Popular, Ayuntamiento de Gijón.

Cantero, C. (2015). El agua en la vida cotidiana. Fuentes, lavaderos y abrevaderos de Asturias. Gijón: Red de Museos Etnográficos de Asturias.

Cantero, C. (2018). Informe Declaración Bien de Interés Cultural de carácter inmaterial. Sidros y comedies.

Cantero, C. (2019). Documento-marco de recomendaciones sobre la gestión de bienes patrimoniales de carácter etnológico: hórreos y paneras. Retrieved from https://www.asturias.es/Asturias/descargas/PDF_TEMAS/Cultura/patrimonio/2 
020_02_11_documento_marco_de_horreos.pdf

Cátedra, M. (1986). Death as a cultural process. The vaqueiros de Alzada, Spain: A dissertation in anthropology. University Microfilms International, Ann Arbor, Michigan.

Cátedra, M. (1988). La muerte y otros mundos: enfermedad, suicidio, muerte y más allá entre los vaqueiros de alzada. Gijón: Júcar.

Cátedra, M. (1989). La vida y el mundo de los vaqueiros de alzada. Madrid: Akal.

Cerra, Y. (1991). Bailes y danzas tradicionales en Asturias. Oviedo: Real Instituto de Estudios Asturianos.

Cerra, Y. (2008). La invención y la recuperación de los bailes tradicionales. In Baille y danza tradicional n'Asturies (pp. 81-102). Gijón: Muséu del Pueblu d'Asturies.

Cerra, Y. (2015). Conservar mientras se da: aldeanas que ofrecen ramos. In N. Herrero (Ed.), Los lugares de la tradición. Antropología social y cultural (pp. 77108). Oviedo: Real Instituto de Estudios Asturianos.

Cerra, Y. (2017). Memoria para la incoación de expediente de declaración de bien de interés cultural de carácter inmaterial del corri-corri.

Cortes-Vazquez, J. A. (2020). In the name of the people: The populist redefinition of nature conservation in post-crisis Spain. Geoforum, 108, 110-118. https://doi.org/10.1016/j.geoforum.2019.12.004

Devillard, M.-J. (1997). «Yé una cadena». Minería y estrategias de reproducción social. Antropología, Revista de Pensamiento Antropológico y Estudios Etnográficos, 13, 103-119.

Devillard, M.-J. (2002). Crisis, realidades y representaciones des espacio minero langreano en vísperas del siglo XXI. In J. L. García (Ed.), Los últimos mineros. Un estudio antropológico sobre la minería en España (pp. 1-53). Madrid: Centro de Investigaciones Sociológicas.

Eriksen, T. H. (2016). Overheating: The World Since 1991. History and Anthropology, 27(5), 469-487. https://doi.org/10.1080/02757206.2016.1218865

Fernández, J. W. (1996). Campos léxicos y vida cultural n'Asturies. Uviéu: Academia de la Llingua Asturiana. 
Fernández, J. W. (2009). El carácter asturiano y otros enigmas e improntas de las identidades hispánicas. Gijón: Muséu del Pueblu d’Asturies.

Fernández, J. W., Cátedra, M., \& García, J. L. (2016). Los inicios de la antropología en Asturias. Tres testimonios autobiográficos. Gijón: Red de Museos Etnográficos de Asturias.

Fernández, J. W., \& Velasco, H. M. (2011). En el dominio del tropo: imaginación figurativa y vida social en España. Madrid: Universidad Nacional de Educación a Distancia.

García, A. (1988). Los vaqueiros de alzada de Asturias. Un estudio históricoantropológico. Oviedo: Servicio de Publicaciones de la Consejería de Educación, Cultura y Deportes.

García, A. (2008). Antropología de Asturias I. La cultura tradicional, patrimonio de futuro. Oviedo: KRK.

García, A. (2011). Antropología de Asturias II. El cambio: la imagen invertida del otro. Oviedo: KRK.

García, J. L. (1976). Antropología del territorio. Madrid: Taller de Ediciones Josefina Betancor.

García, J. L. (1996). Prácticas paternalistas. Un estudio antropológico sobre los mineros asturianos. Barcelona: Ariel.

García, J. L. (2002). Mineros alleranos: de los diferentes segmentos sociales a la construcción de la comunidad. In J. L. García (Ed.), Los últimos mineros. Un estudio antropológico sobre la minería en España (pp. 55-110). Madrid: Centro de Investigaciones Sociológicas.

García, J. L. (2009). La intervención política y la construcción de las categorías colectivas. La prejubilación de los mineros como problema social. In J. L. García (Ed.), Prejubilados españoles. Ajustes y resistencias ante las políticas públicas (pp. 23-65). Buenos Aires: Miño y Dávila.

García, J. L., Velasco, H., \& López Coira, M. (1991). Rituales y proceso social: estudio comparativo en cinco zonas españolas. Madrid: Ministerio de cultura.

Gómez, E. (1990). Vida tradicional y proceso de cambio en un valle del oriente 
asturiano: estudio antropológico del Valle de Ardisana. Oviedo: Universidad de Oviedo.

Gómez, E. (1993). Las mascaradas de invierno en Asturias. Una perspectiva antropológica. Oviedo: Real Instituto de Estudios Asturianos.

González-Quevedo, R. (2002a). Antropología social y cultural de Asturias. Oviedo: Madú Ediciones.

González-Quevedo, R. (2002b). Aspeutos de la muerte n'Asturies. Oviedo: Madú Ediciones.

González-Quevedo, R. (2010). La cultura asturiana. Oviedo: Ediciones CH.

Graña, A., \& López, J. (1983). Hórreos y paneras del concejo de Allande Asturias: evolución y motivos decorativos. Oviedo: Biblioteca Popular Asturiana.

Graña, A., \& López, J. (2007). Los teitos en Asturias: un estudio sobre la arquitectura con cubierta vegetal. Gijón: Red de Museos Etnográficos de Asturias.

Herrero, N. (2014). Las glorietas para la Sacramental de Naves (Asturias): marcadores rituales del territorio. In J. L. García, M. Cátedra, \& M.-J. Devillard (Eds.), Saberes culturales. Homenaje a José Luis García (pp. 545-566). Barcelona: Bellaterra.

Hidalgo, A. (2017). Márgenes y sobras oníricas de la producción de Alberto Cardín (1948-1992). Eikasia, Revista de Filosofía, 74, 143-236. Retrieved from http://revistadefilosofia.com/74-07.pdf

Lellep, R. (1990). A Simple Matter of Salt: An Ethnography of Nutritional Deficiency in Spain. n.d.: University of California Press.

López, J. (1994). Las abejas, la miel y la cera en la sociedad tradicional asturiana. Oviedo: Real Instituto de Estudios Asturianos.

López, J., Quirós, F., \& Graña, A. (1998). Ferrerías, mazos y fraguas en Asturias: documentación para el estudio de la industria tradicional del hierro en el occidente de Asturias. Servicio de Publicaciones del Principado de Asturias.

Lorenzi, E. (2004). Leche y folklore. La incorporación a la economía de mercado y la transformación de la sociedad campesina en Tapia de Casariego. In R. Valdés \& E. Lorenzi (Eds.), ¿Bótoche unha mao?La evolución de las relaciones de 
reciprocidad campesinas en Tapia de Casariego (pp. 85-214). Gijón: Fundación Municipal de Cultura, Educación y Universidad Popular.

Mintz, S. W. (1998). The Localization of Anthropological Practice. Critique of Anthropology, 18(2), 117-133. https://doi.org/10.1177/0308275X9801800201

Molina, J. L. (2011). Ramón Valdés del Toro, Catedrático de Antropología Social y Cultural (1930-2011). Revista de Antropología Social, 20, 331-332.

Moraga, J. (2010). Chinos en Asturias. La reciprocidad en el imperio del cálculo. Gijón: Muséu del Pueblu d'Asturies.

Narotzky, S. (2001). La antropología de los pueblos de España. Historia, Cultura y Lugar. Barcelona: Icaria editorial, Institut Català d'Antropologia.

Pérez-Sutil, R. (2019). Cabranes: de la casería a la casa de huéspedes. Gijón: Red de Museos Etnográficos de Asturias.

Sánchez, J. O. (1992). Ecología y estrategias sociales de los pescadores de Cudillero. Madrid: Siglo Veintiuno: Ministerio de Cultura. Dirección General de Bellas Artes y Archivos.

Sánchez, J. O. (2004). Trabajo, política e ideología en una cuenca minera. Madrid: Siglo Veintiuno.

Santoveña, F. (2013). Vestidos de asturianos. Ciento cincuenta años de fotografía e indumentaria en Asturias. Gijón: Muséu del Pueblu d'Asturies/ Fundación Municipal de Cultura, Educación y Universidad Popular del Ayuntamiento de Gijón.

Santoveña, F. (2018). Traje tradicional, indumentaria popular y construcción del cuerpo en Asturias (1860-1920). Gijón: Muséu del Pueblu d'Asturies/ Fundación Municipal de Cultura, Educación y Universidad Popular del Ayuntamiento de Gijón.

Tsing, A. L. (2005). Friction: An ethnography of global connection. In Friction: An Ethnography of Global Connection. Princeton: Princeton University Press.

Valdés, R. (1976). Ecología y trabajo, fiestas y dieta en un concejo del occidente astur. In C. Lisón (Ed.), Temas de Antropología española (pp. 263-345). Madrid: Akal. 
Viyao Valdés, P., \& Martín-Ayuso Navarro, R. (2007). Dos estudios etnográficos sobre el oriente de Asturias [1920-1921]. Gijón: Red de Museos Etnográficos de Asturias.

Wimmer, A., \& Glick Schiller, N. (2002). Methodological Nationalism and Beyond: Nation-state Building, Migration and the Social Sciences. Global Networks, 2(4), 301-334. https://doi.org/10.1111/1471-0374.00043 